\title{
THE RADIUS OF UNIVALENCE OF CERTAIN ANALYTIC FUNCTIONS. II
}

\section{THOMAS H. MACGREGOR}

1. Introduction. Throughout this paper suppose that $f(z)=z$ $+a_{2} z^{2}+\cdots$ is analytic for $|z|<1$ and $g(z)=z+b_{2} z^{2}+\cdots$ is analytic and univalent for $|z|<1$. Recently [3] the author considered the problem: what is the radius of univalence of the family of functions $f(z)$ which satisfy $\operatorname{Re}\{f(z) / g(z)\}>0$ for $|z|<1$ ? This problem was solved for each of the subfamilies associated with the cases $g(z) \equiv z, g(z)$ is starlike for $|z|<1$, and $g(z)$ is convex for $|z|<1$. Only an estimate was obtained for the radius of univalence of the whole family.

In this paper we consider functions $f(z)$ which satisfy $|f(z) / g(z)-1|$ $<1$ for $|z|<1$. The radius of univalence of this family of functions is determined. Also, we find the radius of univalence and starlikeness for the subfamilies associated with each of the cases: $g(z) \equiv z, g(z)$ is starlike for $|z|<1, g(z)$ is convex for $|z|<1$.

2. THEOREM 1. If $f(z)=z+a_{2} z^{2}+\cdots$ is analytic and satisfies $|f(z) / z-1|<1$ for $|z|<1$ then $f(z)$ is univalent and starlike for $|z|$ $<1 / 2$.

Proof. The function $F(z)=f(z) / z-1$ is analytic and satisfies $|F(z)|<1$ for $|z|<1$ and $F(0)=0$. Thus $F(z)=z \phi(z)$ where $\phi(z)$ is analytic for $|z|<1$ and $|\phi(z)| \leqq 1$. For such functions we have

$$
\left|\phi^{\prime}(z)\right| \leqq \frac{1-|\phi(z)|^{2}}{1-|z|^{2}}
$$

[1, p. 18]. Expressing $f(z)$ and $f^{\prime}(z)$ in terms of $\phi(z)$, we obtain

$$
\frac{z f^{\prime}(z)}{f(z)}=1+z \frac{\phi(z)+z \phi^{\prime}(z)}{1+z \phi(z)} .
$$

Using (1) we obtain

$$
\begin{aligned}
\left|\frac{\phi(z)+z \phi^{\prime}(z)}{1+z \phi(z)}\right| & \leqq \frac{|\phi(z)|+|z| \frac{1-|\phi(z)|^{2}}{1-|z|^{2}}}{1-|z||\phi(z)|} \\
& =\frac{|\phi(z)|+|z|}{1-|z|^{2}} \leqq \frac{1+|z|}{1-|z|^{2}}=\frac{1}{1-|z|},
\end{aligned}
$$

Received by the editors March 16, 1962. 


$$
\left|\frac{\phi(z)+z \phi^{\prime}(z)}{1+z \phi(z)}\right| \leqq \frac{1}{1-|z|} .
$$

From (2) and (3) we obtain

$$
\left|\frac{z f^{\prime}(z)}{f(z)}-1\right| \leqq \frac{|z|}{1-|z|}
$$

If $|z|<1 / 2$ then $\left|z f^{\prime}(z) / f(z)-1\right|<1$ so that, in particular, $\operatorname{Re}\left\{z f^{\prime}(z) / f(z)\right\}>0$ for $|z|<1 / 2$. This proves the theorem since the condition $\operatorname{Re}\left\{z f^{\prime}(z) / f(z)\right\}>0$ for $|z|<r$ is necessary and sufficient for $f(z)$ to be univalent for $|z|<r$ and map that circle onto a domain which is starlike with respect to the origin.

The function $f(z)=z+z^{2}$ satisfies the hypotheses of this theorem but is univalent in no circle $|z|<r$ with $r>1 / 2$ since its derivative vanishes at $z=-1 / 2$.

THEOREM 2. Suppose that $f(z)=z+a_{2} z^{2}+\cdots$ is analytic for $|z|<1$ and $g(z)=z+b_{2} z^{2}+\cdots$ is analytic and univalent for $|z|<1$. If $|f(z) / g(z)-1|<1$ for $|z|<1$ then $f(z)$ is univalent for $|z|<1 / 3$.

Proof. The function $\phi(z)=f(z) / g(z)-1$ is analytic for $|z|<1$ and satisfies $|\phi(z)|<1, \phi(0)=0$. To show that $f(z)$ is univalent in $|z| \leqq r$ it suffices to prove univalence on $|z|=r$. Let $z_{1} \neq z_{2},\left|z_{1}\right|=\left|z_{2}\right|=r$. Then $f\left(z_{1}\right)=f\left(z_{2}\right)$ can be written

$$
\frac{g\left(z_{2}\right)-g\left(z_{1}\right)}{g\left(z_{1}\right)\left(z_{2}-z_{1}\right)}=-\frac{\phi\left(z_{2}\right)-\phi\left(z_{1}\right)}{\left(1+\phi\left(z_{2}\right)\right)\left(z_{2}-z_{1}\right)} .
$$

Thus, $f(z)$ will be univalent in $|z| \leqq r$ if we prove

$$
\left|\frac{g\left(z_{2}\right)-g\left(z_{1}\right)}{g\left(z_{1}\right)\left(z_{2}-z_{1}\right)}\right|>\left|\frac{\phi\left(z_{2}\right)-\phi\left(z_{1}\right)}{\left(1+\phi\left(z_{2}\right)\right)\left(z_{2}-z_{1}\right)}\right| \text {. }
$$

Since $|\phi(z)|<1$ for $|z|<1$ and $\phi(0)=0$ we have $\left|\phi^{\prime}(z)\right| \leqq 1$ for $|z| \leqq \sqrt{2}-1\left[1\right.$, p. 19]. From the representation $\phi\left(z_{2}\right)-\phi\left(z_{1}\right)$ $=\int_{z_{1}}^{z_{2}} \phi^{\prime}(z) d z$ where the path of integration is the line segment from $z_{1}$ to $z_{2}$ the estimate for $\left|\phi^{\prime}(z)\right|$ implies $\left|\phi\left(z_{2}\right)-\phi\left(z_{1}\right)\right| \leqq\left|z_{2}-z_{1}\right|$ for $r \leqq \sqrt{2}-1$. From Schwarz's lemma we also have $\left|\phi\left(z_{2}\right)\right| \leqq\left|z_{2}\right|=r$. Thus,

$$
\left|\frac{\phi\left(z_{2}\right)-\phi\left(z_{1}\right)}{\left(1+\phi\left(z_{2}\right)\right)\left(z_{2}-z_{1}\right)}\right| \leqq \frac{1}{1-r} \quad \text { for } r \leqq \sqrt{2}-1 \text {. }
$$

Since $g(z)=z+b_{2} z^{2}+\cdots$ is analytic and univalent for $|z|<1$ 


$$
\left|\frac{g\left(z_{2}\right)-g\left(z_{1}\right)}{z_{2}-z_{1}}\right| \geqq\left|g\left(z_{1}\right) g\left(z_{2}\right)\right| \frac{1-r^{2}}{r^{2}}
$$

[2]. Using this estimate and the distortion theorem $|g(z)|$ $\geqq|z| /(1+|z|)^{2}$ we obtain

$$
\left|\frac{g\left(z_{2}\right)-g\left(z_{1}\right)}{g\left(z_{1}\right)\left(z_{2}-z_{1}\right)}\right| \geqq \frac{1-r}{r(1+r)} .
$$

From (4) and (5) it follows that $f(z)$ is univalent in $|z| \leqq r$ if $(1-r) / r(1+r)>1 /(1-r)$ and $r \leqq \sqrt{2}-1$. Since $(1-r) / r(1+r)$ $>1 /(1-r)$ is equivalent to $r<1 / 3$ we have shown that $f(z)$ is univalent in $|z|<1 / 3$.

The function $f(z)=\left(z+z^{2}\right) /(1-z)^{2}$ satisfies the hypotheses of this theorem with $g(z)=z /(1-z)^{2}$. This $f(z)$ is not univalent in $|z|<r$ if $r>1 / 3$ since its derivative vanishes at $z=-1 / 3$.

Theorem 3. Suppose that $f(z)=z+a_{2} z^{2}+\cdots$ is analytic for $|z|<1$ and $g(z)=z+b_{2} z^{2}+\cdots$ is analytic, univalent and starlike for $|z|<1$. If $|f(z) / g(z)-1|<1$ for $|z|<1$ then $f(z)$ is univalent and starlike for $|z|<1 / 3$.

Proof. We can write $f(z) / g(z)-1=z \phi(z)$ where $\phi(z)$ is analytic and satisfies $|\phi(z)| \leqq 1$ for $|z|<1$. Expressing $f(z)$ and $f^{\prime}(z)$ in terms of $g(z)$ and $\phi(z)$ yields

$$
\frac{z f^{\prime}(z)}{f(z)}=\frac{z g^{\prime}(z)}{g(z)}+z \frac{\phi(z)+z \phi^{\prime}(z)}{1+z \phi(z)} .
$$

$\left(z f^{\prime}(z) / f(z)\right.$ is analytic in $|z|<1$ since the hypotheses imply that $f(z)$ does not vanish in $|z|<1$ except for the simple zero at $z=0$.)

Since $g(z)$ is starlike $\operatorname{Re}\left\{z g^{\prime}(z) / g(z)\right\}>0$ for $|z|<1$ and, therefore,

$$
\operatorname{Re}\left\{\frac{z g^{\prime}(z)}{g(z)}\right\} \geqq \frac{1-|z|}{1+|z|}
$$

[5, p. 140]. Applying this estimate and also (3) to (6) gives

$$
\operatorname{Re}\left\{\frac{z f^{\prime}(z)}{f(z)}\right\} \geqq \frac{1-|z|}{1+|z|}-\frac{|z|}{1-|z|}=\frac{1-3|z|}{1-|z|^{2}}
$$

Therefore, if $|z|<1 / 3$ then $\operatorname{Re}\left\{z f^{\prime}(z) / f(z)\right\}>0$. This proves the theorem.

The function $f(z)=\left(z+z^{2}\right) /(1-z)^{2}$ is an extremal function for this theorem as well as for Theorem 2 since $g(z)=z /(1-z)^{2}$ maps $|z|<1$ onto a starlike domain. 
TheOREM 4. Suppose that $f(z)=z+a_{2} z^{2}+\cdots$ is analytic for $|z|<1$ and $g(z)=z+b_{2} z^{2}+\cdots$ is analytic, univalent and convex for $|z|<1$. If $|f(z) / g(z)-1|<1$ for $|z|<1$ then $f(z)$ is univalent and starlike for $|z|<\sqrt{2}-1$.

Proof. Since $g(z)$ is univalent and convex for $|z|<1$ we have $\operatorname{Re}\left\{z g^{\prime}(z) / g(z)\right\}>1 / 2$ for $|z|<1$ and, therefore, $\operatorname{Re}\left\{z g^{\prime}(z) / g(z)\right\}$ $\geqq 1 /(1+|z|)[4 ; 6]$. Proceeding exactly as in the proof of Theorem 3 this improved estimate on $\operatorname{Re}\left\{z g^{\prime}(z) / g(z)\right\}$ gives

$$
\operatorname{Re}\left\{\frac{z f^{\prime}(z)}{f(z)}\right\} \geqq \frac{1}{1+|z|}-\frac{|z|}{1-|z|}=\frac{1-2|z|-|z|^{2}}{1-|z|^{2}} .
$$

If $|z|<\sqrt{2}-1$ then $1-2|z|-|z|^{2}>0$. Therefore, $f(z)$ is univalent and starlike in $|z|<\sqrt{2}-1$.

The function $f(z)=\left(z+z^{2}\right) /(1-z)$ satisfies the hypotheses of this theorem with $g(z)=z /(1-z)$. Since the derivative of this $f(z)$ vanishes at $z=1-\sqrt{2}$ it is not univalent in $|z|<r$ if $r>\sqrt{2}-1$.

\section{REFERENCES}

1. C. Carathéodory, Theory of functions, Vol. 2, Chelsea, New York, 1954.

2. G. M. Golusin, On distortion theorems and coefficients of univalent functions, Mat. Sb. (N.S.) 19 (61) (1946), 183-202. (Russian, English summary)

3. T. MacGregor, The radius of univalence of certain analytic functions, Proc. Amer. Math. Soc. 14 (1963), 514-520.

4. A. Marx, Untersuchungen über schlichte Abbildungen, Math. Ann. 107 (1932), $40-67$.

5. G. Polya and G. Szegö, Aufgaben und Lehrsätze aus der Analysis, Vol. 1, Springer, Berlin, 1954.

6. E. Strohhäcker, Beiträge zur Theorie der schlichten Funktionen, Math. Z. 37 (1933), 356-380.

\section{Lafayette College}

